\title{
5-Aza-2'-deoxycytidine induces cytotoxicity in BGC-823 cells via DNA methyltransferase 1 and 3a independent of p53 status
}

\author{
JUAN LIU $^{1 *}$, YAN ZHANG $^{2 *}$, YI-SHAN XIE ${ }^{3}$, FU-LIANG WANG ${ }^{1}$, \\ LI-JUN ZHANG ${ }^{1}$, TAO DENG ${ }^{2}$ and HE-SHENG LUO ${ }^{2}$ \\ Departments of ${ }^{1}$ Geriatrics, ${ }^{2}$ Gastroenterology and ${ }^{3}$ Oncology, Renmin Hospital of \\ Wuhan University, Wuchang District, Wuhan 430060, P.R. China
}

Received January 31, 2012; Accepted April 27, 2012

DOI: $10.3892 /$ or.2012.1838

\begin{abstract}
The DNA methylation inhibitor 5-aza-2'-deoxycytidine (5-Aza-CdR) has therapeutic value for the treatment of cancer. However, the mechanism by which 5-Aza-CdR induces antineoplastic activity is not clear. The efficacy of 5-Aza-CdR on the contribution of gene reactivation by demethylation and enzyme-DNA adduct formation is an important unresolved question. The aim of this study was to explore the mechanism of the effect of 5-Aza-CdR on human gastric cancer growth. Human BGC-823 cells were treated with different concentrations of 5-Aza-CdR for different durations. Cell viability, DNA damage and gene expression were determined. The results showed that $5-\mathrm{Aza}-\mathrm{CdR}$ at low concentrations induced inhibition of gastric cancer BGC-823 cell proliferation as well as increased apoptosis caused by DNA damage. For the first time, we demonstrated that 5-Aza-CdR-induced cytotoxicity against BGC-823 cells was predominantly regulated via upregulation of DNA methyltransferase 1, 3a and partially via reactivation of RUNX3, which was independent of p53 status and its ability to activate $\mathrm{p} 21^{\mathrm{Waf} 1 / \mathrm{Cip} 1}$ expression. To our knowledge, this is the first demonstration of p53-independent 5-Aza-CdR action on DNA methyltransferases and demethylation. These results strongly provide the preclinical rationale for the clinical evaluation of 5-Aza-CdR to improve patient outcome in gastric cancer.
\end{abstract}

\section{Introduction}

DNA methylation and related modulation of gene expression contribute to the development of malignancies $(1,2)$.

Correspondence to: Professor He-Sheng Luo or Professor Tao Deng, Department of Gastroenterology, Renmin Hospital of Wuhan University, 238 Jiefang Road, Wuchang District, Wuhan 430060, P.R. China

E-mail: luo20111016@sina.com

\section{${ }^{*}$ Contributed equally}

Key words: p53, apoptosis, runt-related transcription factor 3, 5-aza-2'-deoxycytidine, DNA methyltransferase
Specifically, methylation of $\mathrm{CpG}$ dinucleotides in promoter regions has been associated with transcriptional silencing of tumor suppressor genes, suggesting DNA methylation as a target for novel therapeutics (3). 5-Azacytidine and 5-Aza-2'deoxycytidine (5-Aza-CdR) belong to a class of cytosine analogues, which are developed as inhibitors of DNA methylation. Both analogues have been shown to have significant cytotoxic and antineoplastic activities in many experimental tumors $(3,4)$. 5-Aza-CdR, however, is reported to be noncarcinogenic. It incorporates into only DNA but not RNA or proteins (5). Additionally, considerable evidence shows that 5-Aza-CdR has more potent therapeutic effects than 5-Azacytidine in cell culture and animal models of human cancers.

5-Azacytidine is the only DNA methyltransferase (DNMT) inhibitor (MTI) approved by the Food and Drug Administration for treating myelodysplastic syndromes and chronic myelomonocytic leukemia (6). Recently, several clinical trials of 5-Aza-CdR have been reported, including a phase II study of 5-Aza-CdR in patients with metastatic prostate cancer and a phase III study of 5-Aza-CdR (Decitabine) in patients with myelodysplasia (7). Clinical trials evaluating 5-Aza-CdR as a cancer chemotherapeutic have shown promise for the treatment of leukemia, but less effective against solid tumors.

5-Aza-CdR binds DNMT in an irreversible, covalent manner, thus sequestering DNMT enzyme activity and preventing maintenance of the DNA methylation state (8). Consequently, silenced genes are demethylated and are re-expressed. Two major non-mutually exclusive mechanisms of its tumor cytotoxicity have been proposed. One states that 5-Aza-CdR demethylates cellular DNA, with reactivation of silenced genes; and the other states that 5-Aza-CdR induces DNA damage due to the formation of irreversible, covalent enzyme-DNA adducts (9). However, the relative contribution of gene reactivation and enzyme-DNA adduct formation to the efficacy and toxicity of 5-Aza-CdR in vivo remains unresolved.

As one of the major causes of cancer death, gastric cancer remains threatening around the world, and most patients in advanced stages need chemotherapy. To date, however, the effects of 5-Aza-CdR and mechanisms against gastric cancer have not been completely characterized. Here, we show that 5-Aza-CdR is cytotoxic against human gastric cancer 
BGC-823 cell growth. Mechanistic studies demonstrated that 5-Aza-CdR induces DNA damage and causes apoptosis of gastric cancer cells. These responses were mediated mainly through upregulation of DNMT and partially through demethylation of runt-related transcription factor 3 (RUNX3), which was independent of $\mathrm{p} 53$ status and its ability to activate $\mathrm{p} 21^{\text {Wafl/Cipl }}$ expression. Taken together, these studies provide the preclinical rationale for the clinical evaluation of 5-Aza$\mathrm{CdR}$ to improve patient outcome in gastric cancer.

\section{Materials and methods}

Cells and treatments. Human lung cancer cell line A549 and the gastric cancer cell line BGC-823 were obtained from the China Center for Type Culture Collection (CCTCC). Both cell lines were grown in Dulbecco's modified Eagle's medium (DMEM) containing $10 \%$ fetal bovine serum at $37^{\circ} \mathrm{C}$ in a humidified atmosphere with $5 \% \mathrm{CO}_{2}$. For treatment with 5-Aza-CdR (Sigma), cells were exposed to a single dose of $0.01-100 \mu \mathrm{M}$ of drug. 5-Aza-CdR was dissolved in phosphate-buffered saline (PBS) and fresh medium containing 5-Aza-CdR was added every $24 \mathrm{~h}$.

MTT assay. Cell proliferation was measured using the MTT assay. Cells were plated in triplicate at $1 \times 10^{3}$ cells/well in 96-well plates, cultured as previously described, and treated with different concentrations of 5-Aza-CdR or z-VAD-fmk (Beyotime Institute of Biotechnology) for indicated times respectively. Twenty microliters of $5 \mathrm{mg} / \mathrm{ml}$ of MTT (Amresco) was then added into each well and the cells were cultured at $37^{\circ} \mathrm{C}$ for an additional 4-6 h. The resulting formazan crystals were solubilized by the addition of $150 \mu \mathrm{l}$ of DMSO to each well. The optical density (OD) level under $570 \mathrm{~nm}$ was measured and the percentage of cell viability was calculated using the following formula: percentage of cell viability = (absorbance experimental well - absorbance $\left._{\text {blank }}\right) /\left(\right.$ absorbance $_{\text {untreated }}$ control well - absorbance $_{\text {blank }}$ ) x $100 \%$.

Flow cytometric analysis of DNA content. Cells were seeded into 6-well plates at a density of 4 to $5 \times 10^{5}$ cells/well. After $72 \mathrm{~h}$ of treatment with $0.5,1$ and $5 \mu \mathrm{M} 5$-Aza-CdR, cells were washed with PBS, permeabilized with $70 \%$ ethanol overnight. The next day, ethanol was removed and cells were incubated for $15-20 \mathrm{~min}$ at $37^{\circ} \mathrm{C}$ with $1 \mathrm{ml} \mathrm{PI}$ solution $(0.1 \%$ Triton X-100, $50 \mu \mathrm{g}$ PI and $200 \mu \mathrm{g}$ RNase A). Distribution of cell cycle phases with different DNA contents was determined using a flow cytometer (Beckman Coulter, Miami, FL, USA).

Annexin V staining. Cells (5 to $7 \times 10^{5}$ ) were seeded into 6-well plates and treated with $0.5,1$ and $5 \mu \mathrm{M}$ of 5 -Aza-CdR for $48 \mathrm{~h}$. The cells were then trypsinized and washed in PBS. Annexin V staining was then performed following the manufacturer's instructions of the Annexin V Staining kit (Multi Sciences Biotech Co., Ltd).

Measurement of caspase-3 activity. Caspase-3 activity in the cell lysate was measured using a colorimetric kit according to the manufacturer's instructions (Beyotime Institute of Biotechnology). The absorbance was determined at $405 \mathrm{~nm}$ and activity of caspase- 3 was determined by calculating the ratio of $\mathrm{OD}$ at $405 \mathrm{~nm}$ of drug treated cells to untreated cells.

Comet assay for detecting DNA strand breaks. The Comet assay was performed as previously described (10). In brief, slides were cleaned with acid and scrapped with $40 \mu 1$ of $0.6 \%$ agarose. Cell suspension $(20 \mu \mathrm{l})$ and $80 \mu \mathrm{l}$ of $1.1 \%$ low-melting agarose were mixed before adding to the first gel layer. The gel layer was covered with a coverslip immediately, and then was kept at $4^{\circ} \mathrm{C}$ for $15 \mathrm{~min}$ to allow it to solidify. After gently removing the coverslip, the slides were immersed in fresh prepared cold lysis solution $\left(2.5 \mathrm{M} \mathrm{NaCl}, 100 \mathrm{mM} \mathrm{Na}_{2}\right.$ EDTA, $10 \mathrm{mM}$ Tris, $\mathrm{pH} 10.0$ ) with $1 \%$ Triton X-100 and $10 \%$ DMSO for at least $1 \mathrm{~h}$ at $4^{\circ} \mathrm{C}$. After electrophoresis in fresh solution (1 mM Na 2 EDTA, $300 \mathrm{mM} \mathrm{NaOH}$, pH 13.0) for $30 \mathrm{~min}$, the slides were placed in Tris buffer (0.4 M Tris, $\mathrm{pH} 7.5$ ) for $15 \mathrm{~min}$ twice. The slides were then stained with $40 \mu \mathrm{l}$ of $0.1 \mathrm{mg} / \mathrm{ml}$ propidium iodide (PI). One hundred randomly selected cells were counted per slide. The images were captured and scored for each sample using an Image Analysis software system (IMI version 1.0). The standard of assessing DNA single strand breaks was based on the percentage of cells with tail and tail length (a distance from DNA head to the end of DNA tail) by visual estimation.

$R N A$ preparation and $R T-P C R$. Total RNA was isolated using TRIzol reagent (Invitrogen) and $1 \mu \mathrm{g}$ of RNA was used as a template for the synthesis of cDNA using the RevertAid ${ }^{\mathrm{TM}}$ first strand cDNA synthesis kit (Fermentas) according to the manufacturer's instructions. Polymerase chain reaction (PCR) analysis was performed in a final volume of $25 \mu \mathrm{l}$ using the PCR Master Mix (Fermentas). Primer sequences and annealing temperatures are indicated in Table I. PCR products were separated on $1.5 \%$ agarose gels, stained with ethidium bromide and photographed.

Western blotting. After cells were cultured with the indicated concentrations of 5-Aza-CdR for the specified times, cells were lysed using RIPA buffer containing protease inhibitors. After normalization for total protein content (50 $\mu \mathrm{g} / \mathrm{lane})$, samples were subjected to $15 \%$ SDS-PAGE and then transferred to nitrocellulose membranes (Bio-Rad Laboratories). After blocking with 5\% non-fat dry milk and $0.1 \%$ Tween-20 in Tris-buffered saline, membranes were incubated with mouse anti-p21 $1^{\text {Waf1/Cip1 } 1}$, 53 (Santa Cruz Biotechnology), goat anti-RUNX3 (R\&D Systems), rabbit anti-caspase-3, $\beta$-tubulin (Santa Cruz Biotechnology). After extensive rinsing with TBST buffer, blots were incubated with HRP-conjugated anti-rabbit, anti-mouse or anti-goat secondary antibodies (Pierce) and developed with the use of an enhanced chemiluminescence system (Millipore) and captured on light-sensitive imaging film (Kodak, Tokyo, Japan).

Statistical analysis. All experiments were repeated in triplicate. Data are presented as means $\pm \mathrm{SD}$. Levels of significance for comparisons between samples were determined using the Student's t-test distribution. Statistical analyses among more than three samples were performed by ANOVA with Tukey's honest significant difference post hoc test applied to significant main effects or interactions (SPSS 11.5 for Windows). 
Table I. Primers and conditions used for RT-PCR.

\begin{tabular}{|c|c|c|c|}
\hline Primers & Sequences & Temperature $\left({ }^{\circ} \mathrm{C}\right)$ & Product length (bp) \\
\hline GAPDH & $\begin{array}{l}\text { F: 5'-ACGGATTTGGTCGTATTGGG-3' } \\
\text { R: 5'-TCCTGGAAGATGGTGATGGG-3' }\end{array}$ & 56 & 211 \\
\hline RUNX3 & $\begin{array}{l}\text { F: 5'-GAAAAGGCGTAAGGGAACTC-3' } \\
\text { R: 5'-ACCTGGGACCAGCTATAACC-3' }\end{array}$ & 56 & 395 \\
\hline $\mathrm{p} 21^{\text {Waf1/Cip1 }}$ & $\begin{array}{l}\text { F: 5'-GTGAGCGATGGAACTTCGACT-3' } \\
\text { R: 5'-CGAGGCACAAGG GTACAAGAC-3' }\end{array}$ & 56 & 229 \\
\hline p53 & $\begin{array}{l}\text { F: 5'-GTCTACCTCCCGCCATAA-3' } \\
\text { R: 5'-CATCTCCCAAACATCCCT-3' }\end{array}$ & 55 & 316 \\
\hline DNMT1 & $\begin{array}{l}\text { F: 5'-CGCTGTATCTAGCAAGGGTCA-3' } \\
\text { R: 5'-TCGAATCTCGCGTAGTCTTG-3' }\end{array}$ & 56 & 313 \\
\hline DNMT3a & $\begin{array}{l}\text { F: 5'-ACCACAGAGGCGGAAATACC-3' } \\
\text { R: 5'-GTCTCCCTGCTGCTAACTGG-3' }\end{array}$ & 56 & 543 \\
\hline DNMT3b & $\begin{array}{l}\text { F: 5'-CAGGAGACCTACCCTCCACA-3' } \\
\text { R: 5'-TTACGTCGTGGCTCCAGTTA-3' }\end{array}$ & 56 & 436 \\
\hline
\end{tabular}

\section{Results}

5-Aza-CdR inhibits BGC-823 cell growth by induction of apoptosis. Human lung cancer A549 cells were used as a control since it is well-established that $5-\mathrm{Aza}-\mathrm{CdR}$ potentially overcomes growth advantages (11). Human BGC-823 cells and A549 cells were exposed to 5-Aza-CdR at different concentrations for $72 \mathrm{~h}$. The cell viability was determined by the MTT assay. A dose-dependent inhibition of cell proliferation was observed in both BGC-823 and A549 cells. As shown in Fig. 1A, BGC cell viability was $76.23 \%$ after $1 \mu \mathrm{M} 5$-AzaCdR treatment, while its viability was only about $25 \%$ after $100 \mu \mathrm{M}$ 5-Aza-CdR treatment.

Thus, $1 \mu \mathrm{M}$ 5-Aza-CdR treatment resulted in cell growth inhibition in both cell lines. Therefore, to determine the duration effect of 5-Aza-CdR on cell viability, BGC-823 cells were treated with $1 \mu \mathrm{M}$ of 5 -Aza-CdR for different durations. As expected, we observed not only a dose-dependent growth suppression, but also a duration-dependent growth suppression in BGC-823 cells (Fig. 1B). BGC-823 cell viability was $90,82,70$ and $38 \%$ after $24,48,72$ and $96 \mathrm{~h}$ of treatment, respectively.

To dissect the mechanism of the anti-proliferative effects of 5-Aza-CdR in BGC-823 cells, we determined whether inhibition in cell viability was associated with specific cell cycle arrest. Exponentially growing BGC-823 cells were treated with $0.5,1$ or $5 \mu \mathrm{M} 5$-Aza-CdR for $72 \mathrm{~h}$. The cells were harvested for flow cytometric analysis of DNA content by PI staining as described in Materials and methods. The results showed that $\mathrm{BGC}-823$ cell cycles were not affected by the presence of 5-Aza-CdR (Fig. 1C).

Next, we aimed to examine whether 5-Aza-CdR could efficiently trigger apoptosis, which led to cytotoxicity against BGC-823 cells. BGC-823 cells were treated with $0.5,1$ and $5 \mu \mathrm{M}$ of $5-\mathrm{Aza}-\mathrm{CdR}$ for $48 \mathrm{~h}$. The cells were subjected to apoptosis analysis using Annexin V staining flow cytometry assay as described in Materials and methods. As shown in Fig. 1D, the data revealed that 5-Aza-CdR treatment increased the proportion of cells positive for Annexin $\mathrm{V}$ from $0.7 \%$ in pretreated cells to $15.5,32.7$ and $49.6 \%$ after 5-Aza-CdR treatment at $0.5,1$ and $5 \mu \mathrm{M}$, respectively. The results strongly suggested that apoptosis rather than necrosis is the mechanism of 5-Aza-CdR induced growth inhibition in BGC-823 cells.

Treatment with 5-Aza-CdR causes DNA damage independent of the activation of the caspase pathway in BGC-823 cells. Since 5-Aza-CdR has been reported to incorporate into DNA and not RNA (5), we evaluated the effect of 5-Aza-CdR on DNA damage. BGC-823 cells were treated with 5-Aza-CdR at $0.5,1$ and $5 \mu \mathrm{M}$ for $72 \mathrm{~h}$. DNA damage was determined using the Comet assay. As shown in Fig. 2A, dose-dependent DNA damage was observed upon 5-Aza-CdR treatment in both BGC-823 cells. Not only more comet cells but also longer DNA tail lengths were observed with increased dosage treatment, indicating more extensive DNA damage at higher concentrations. As described in Materials and methods, the image was scored for the percentage of cells with tail and for DNA tail length. As shown in Fig. 2A, the percentage of cells with tail increased from $5.85 \%$ in untreated cells to 15.68 , 48.20 and $60.97 \%$ in $0.5,1$ and $5 \mu \mathrm{M}$ treated cells, respectively; while the length of DNA tail increased from 12.10 in untreated cells to $36.33,57.87$, and 71.33 in $0.5,1$ and $5 \mu \mathrm{M}$ treated cells, respectively.

To analyze whether caspase-3 was responsible for the recruitment of apoptotic pathways by 5 -Aza-CdR, we performed a colorimetric enzyme assay to examine the activity of caspase- 3 and western blotting to detect the protein level of procaspase- 3 . The results showed that caspase- 3 enzymatic activity was not affected in the 5-Aza-CdR-treated cells (Fig. 2B). The protein level of procaspase-3 was not affected by 5 -Aza-CdR treatment either, as monitored by western blot analysis (Fig. 2C). 

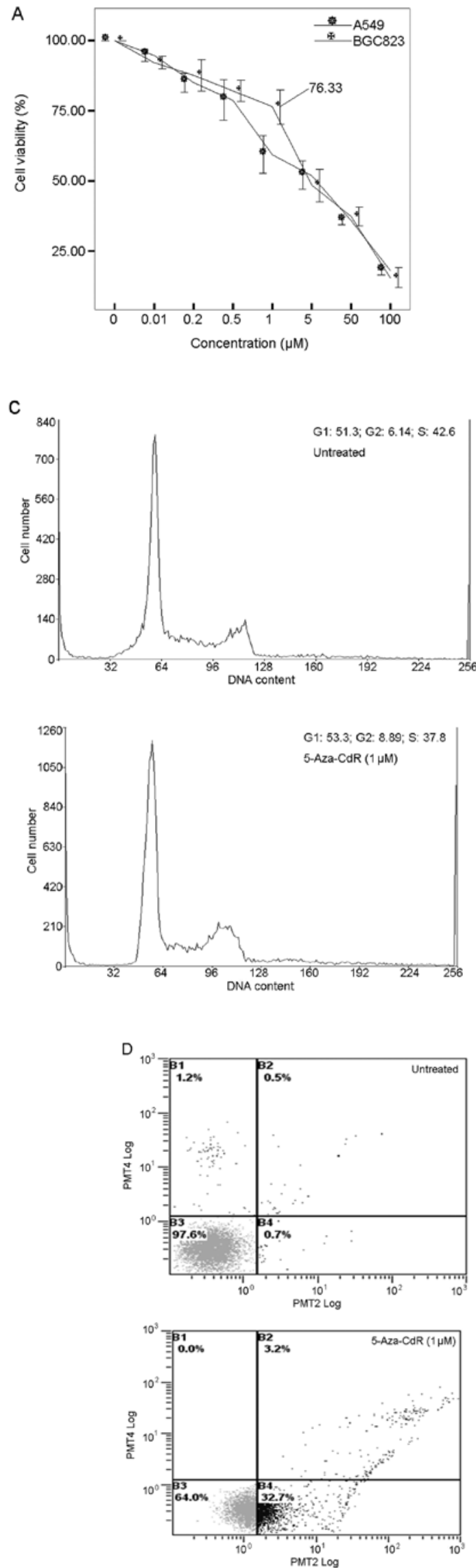
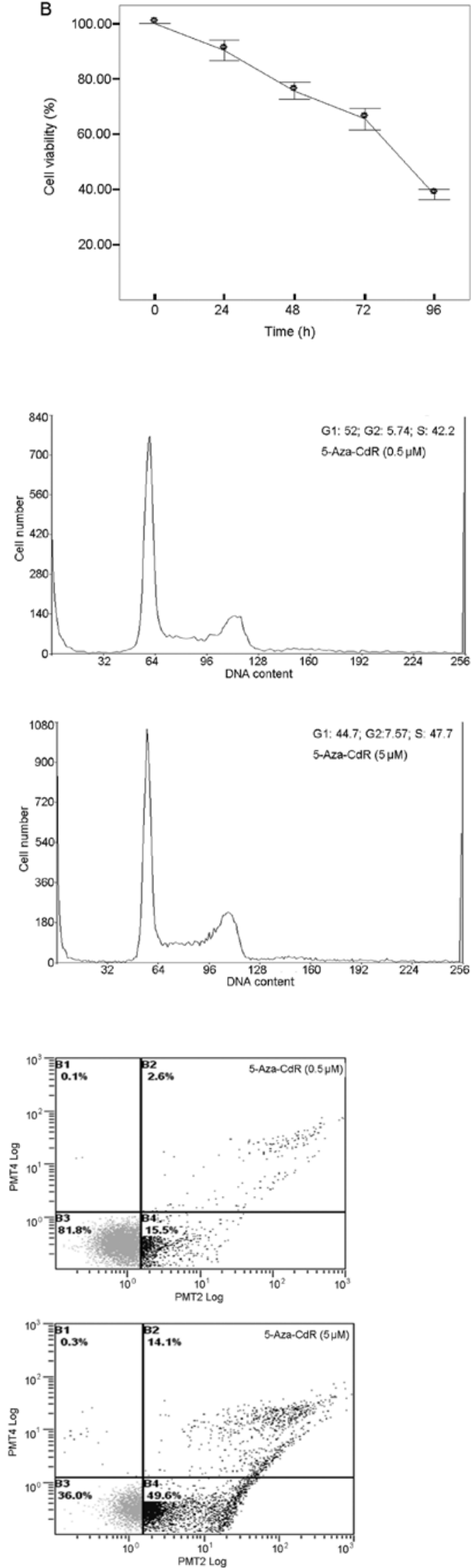

Figure 1.5-Aza-CdR-induced cell proliferation inhibition and apoptosis in A549 and BGC-823 cells. Cell viability was measured with MTT when A549, BGC 823 cells were treated with 5-Aza-CdR at $0-100 \mu \mathrm{M}$ for $72 \mathrm{~h}(\mathrm{~A})$ and at $1 \mu \mathrm{M}$ for different intervals (B). Results are presented as the average of quadruplicate measurements, and the bar is the standard deviation. (C) Effects on cell cycle profiles of BGC-823 cells after treatment with 5-Aza-CdR for $72 \mathrm{~h}$ at indicated concentrations. DNA content was determined by flow cytometric analysis by PI staining. (D) Percentage of apoptotic cells were scored after cells exposure to 5-Aza-CdR at $0,0.5,1$ and $5 \mu \mathrm{M}$ for $48 \mathrm{~h}$ using the Annexin V flow cytometric assay method. 
A
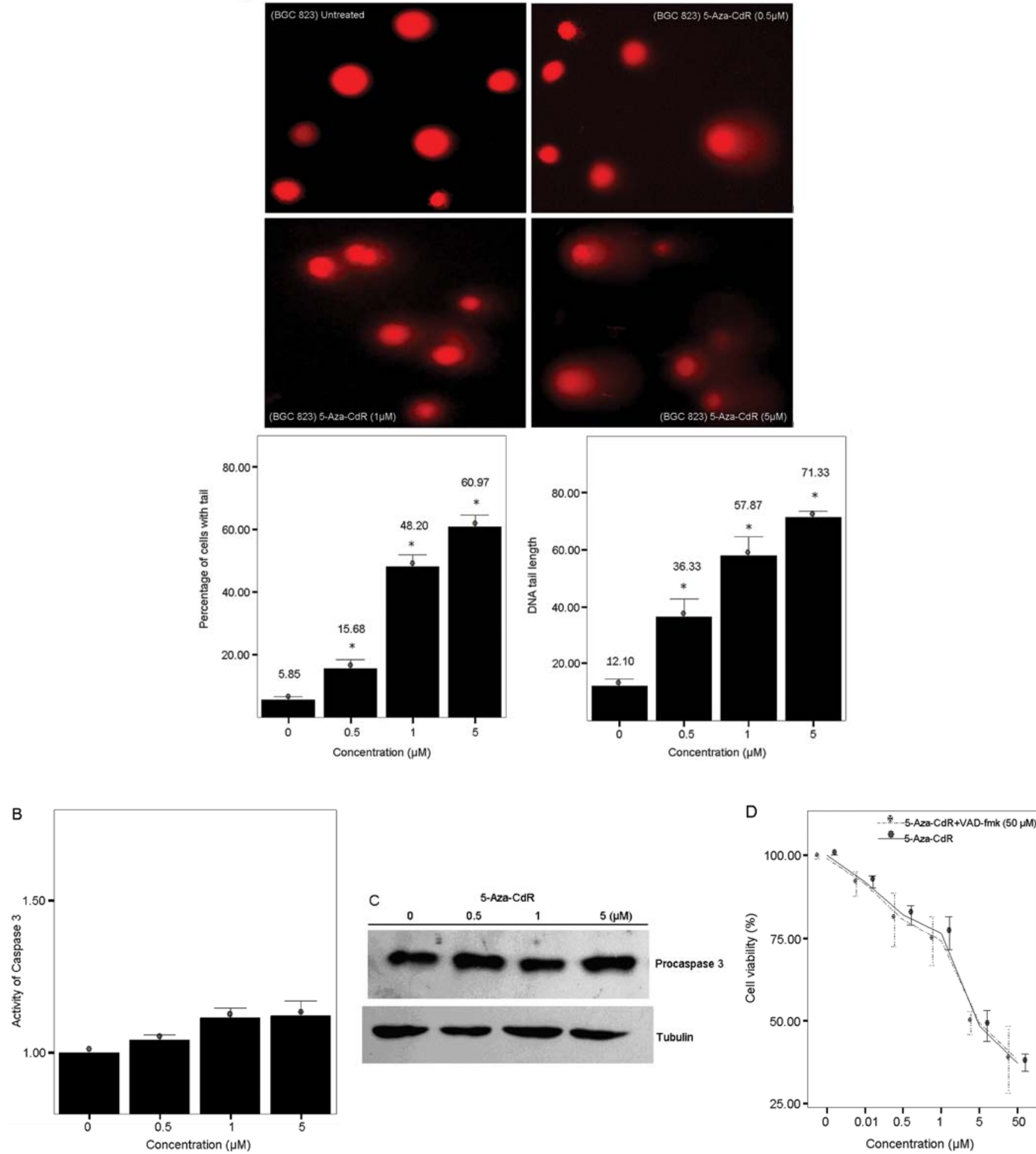

Figure 2. DNA damage and change of caspase- 3 caused by 5 -Aza-CdR in BGC-823 cells. (A) After 5 -Aza-CdR treatment $(0,0.5,1$ and $5 \mu \mathrm{M})$ for $72 \mathrm{~h}, \mathrm{DNA}$ damage in BGC 823-cells were detected using the Comet assay. DNA damage was characterized by the percentage of cells with a Comet tail/100 cells (\%) and the comet tail length (from the center of DNA head to the end of the DNA tail) in BGC-823 cells. Representative pictures of BGC-823 cells are shown. ${ }^{*} \mathrm{P}<0.01$, as compared with respective untreated cells. Bars, mean of triplicate experiments; error bars, SD. (B) Induction of caspase-3 activity level. (C) Expression of procaspase-3 in BGC-823 cells was determined using colorimetric and western blotting methods respectively. (D) The pan-caspase inhibitor, z-VAD-fmk did not significantly alter the cytotoxicity of 5-Aza-CdR-treated BGC-823 cells, as examined by the MTT assay. Results represent the average of 3 independent experiments.

Furthermore, BGC-823 cells were pretreated with the pancaspase inhibitor, $\mathrm{z}-\mathrm{VAD}$-fmk $(5 \mu \mathrm{M})$, and then treated with various concentrations of 5-Aza-CdR for $72 \mathrm{~h}$. Treatment with
z-VAD-fmk did not alter the cytotoxic effect of 5-Aza-CdR, implying that the 5-Aza-CdR-induced apoptosis was independent of the caspase pathway (Fig. 2D). 


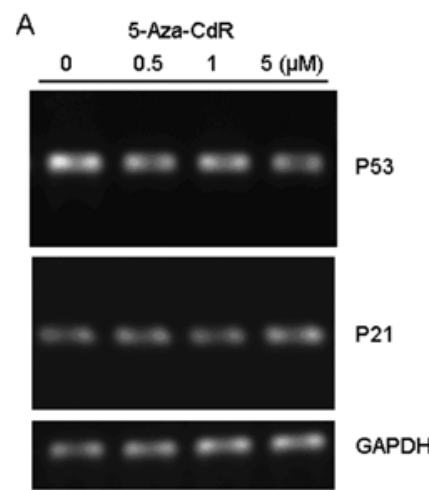

B

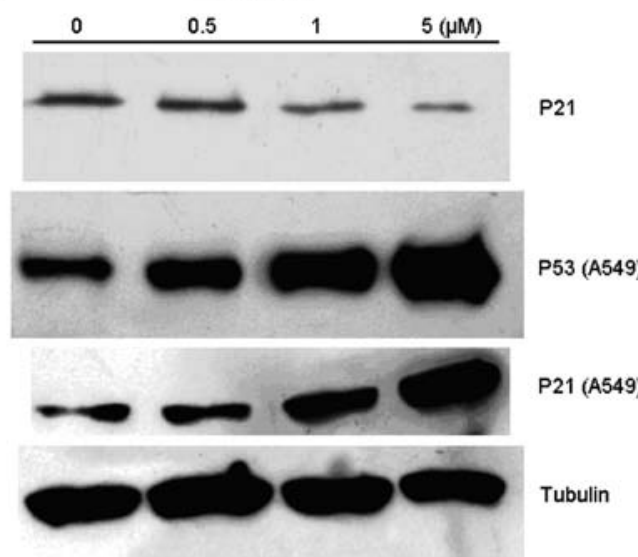

Figure 3. Effects of treatment with 5-Aza-CdR for $72 \mathrm{~h}$ on the expression of $\mathrm{p} 53$ and $\mathrm{p} 21^{\text {Waf1/Cip1 }}$. (A) $\mathrm{p} 53$ or $\mathrm{p} 21^{\text {Wafl/Cip1 }}$ mRNA levels measured by RT-PCR after BGC-823 cells were treated with 5-Aza-CdR at indicated doses. GAPDH was used as an internal control. (B) The protein level of $\mathrm{p} 21^{\text {Waf } 1 / \text { Cip }}$ in BGC-823 cells and $\mathrm{p} 53$ or p $21^{\text {Waf } 1 / \text { Cipl } 1}$ protein levels in A549 cells measured by western blotting. $\beta$-tubulin was used as an internal control.

5-Aza-CdR has no effect on $p 53$ and p21 Wafl/Cipl expression. Because it has been proposed that $\mathrm{p} 53$ has a vital role in growth arrest and apoptosis in response to DNA damage, we explored the p53 expression in response to 5-Aza-CdR treatment in BGC-823 cells. p53 mRNA levels were determined by RT-PCR using total-RNA isolated from BGC-823 cells treated with $0.5,1$ and $5 \mu \mathrm{M} 5$-Aza-CdR for $72 \mathrm{~h}$. The results revealed that p53 mRNA levels were not changed in the presence of 5-Aza-CdR (Fig. 3A). Of interest, the protein expression of p53 was undetectable (data not shown).

It has been well established that the expression of $\mathrm{p} 21^{\mathrm{Waf} 1 / \mathrm{Cip} 1}$ is regulated by p53 (12); therefore, we examined the expression of $\mathrm{p} 21^{\text {Waf1/Cip1 }}$ in BGC-823 cells. $\mathrm{p} 21^{\text {Waf1/Cip1 }}$ gene expression was altered in BGC-823 cells exposed to 5-Aza-CdR (Fig. 3A). As a control, 5-Aza-CdR treatment increased the protein level of $\mathrm{p} 21^{\mathrm{Waf} 1 / \mathrm{Cip} 1}$ along with elevation of $\mathrm{p} 53$ protein levels in a dosedependent manner in A549 cells (Fig. 3B).

5-Aza-CdR induces RUNX3, DNMT1 and DNMT3a gene transcription. 5-Aza-CdR has been reported to exert its cytotoxicity through depleting DNA methyltransferase levels, resulting in DNA hypomethylation (8). We thus studied the expression of RUNX3, a 5-Aza-CdR inducible, methylationregulated target gene. RUNX3 promoter is highly methylated
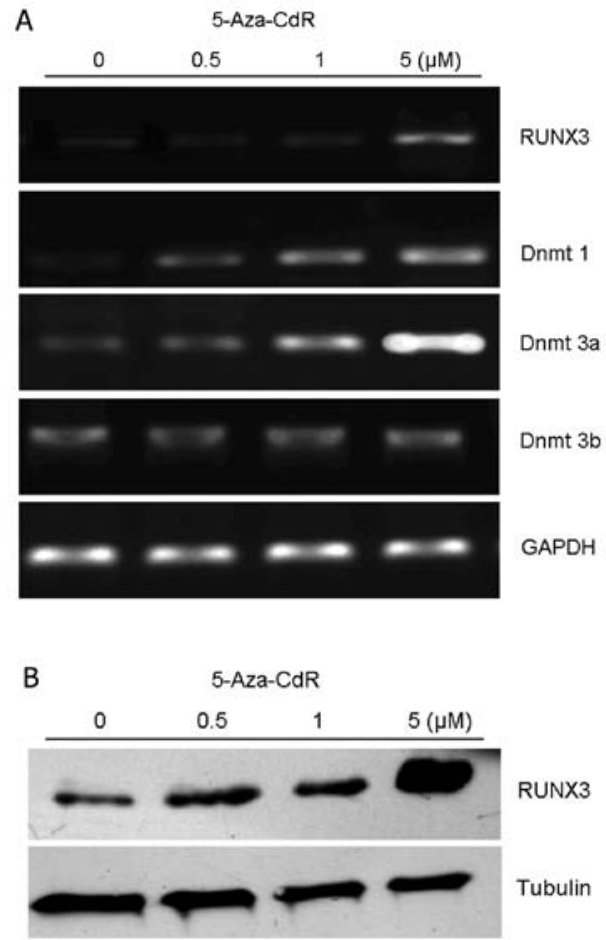

Figure 4. Change of RUNX3 and DNA methyltransferases induced by 5-Aza-CdR. After BGC-823 cells were exposed to 5-Aza-CdR for $72 \mathrm{~h}$, the reactivation of RUNX3 was determined by RT-PCR (A) and western bloting (B). GAPDH and $\beta$-tubulin were used as an internal control.

in gastric cancer cells (13). The RUNX3 protein and mRNA level were determined in BGC-823 cells treated with $0.5,1$ and $5 \mu \mathrm{M} 5$-Aza-CdR for $72 \mathrm{~h}$. As shown in Fig. 4A, it was indicated that RUNX3 mRNA levels were increased in the presence of 5-Aza-CdR at $5 \mu \mathrm{M}$ compared to untreated cells. However, 0.5 and $1 \mu \mathrm{M}$ of 5-Aza-CdR exposure did not affect the levels of RUNX3, consistent with the finding from western blot analysis (Fig. 4B).

Since 5-Aza-CdR is an inhibitor of DNMTs, we analyzed the association between 5-Aza-CdR and DNMT1, DNMT3a, and DNMT3b in BGC-823 cells with RT-PCR analysis. Upon exposure of BGC-823 cells to $0.5 \mu \mathrm{M}$ of 5-Aza-CdR for $72 \mathrm{~h}$, the mRNA levels of DNMT1 and DNMT3a were upregulated in a dose-dependent fashion. The mRNA levels of DNMT3b, however, remained unchanged between drug-treated cells and untreated cells (Fig. 4A).

\section{Discussion}

A number of studies have reported that 5-Aza-CdR treatment decreases cell proliferation and induces apoptosis in tumors, but the mechanisms remain unclear $(14,15)$. In our investigation, we observed that 5-Aza-CdR increased DNA methyltransferease expression and demethylation of RUNX3, independent of the activation of the caspase pathway and the p53/p21 pathway.

From our results, we found that 5-Aza-CdR significantly inhibited BGC-823 cell growth in a dose- and durationdependent manner. Regarding the mechanism underlying the 5-Aza-CdR-iduced inhibition of cell growth, we demonstrated that induction of apoptosis resulted from DNA damage, while 
cell cycle arrest and activation of the caspase pathway were not apparent. This finding is not in line with a previous report that 5-Aza-CdR led to a caspase-dependent induction of apoptosis (16), suggesting different apoptotic pathways rather than caspase may be activated by 5 -Aza-CdR $(17,18,25)$.

p53, a tumor suppressor, stands at the crossroads of cellular responses to various stresses. Both the quantity and activity of p53 are greatly increased in response to DNA damage. Data from PCR and western blotting in BGC-823 cells support the model that 5-Aza-CdR-induced cytoxicity is part of a classical response to DNA damage through induction of the expression of p53 post-translationally rather than hypomethylation at the p53 promoter (19). However, we did not detect any expression changes of p53 mRNA levels in 5-Aza-CdR-treated BGC-823 cells. This may be due to gene mutation (20), as p53 protein levels were undetectable in untreated and treated BGC-823 cells.

Actually, some investigators have indicated that the cell lines with wild-type p53 are more sensitive to 5-Aza-CdR than that of mutant p53 $(11,21)$. However, Nieto et al (22) have shown that p53 null mouse embryonic fibroblast (MEF) cells are more sensitive to 5-Aza-CdR than p53-positive cells. In line with this report, when the null expression of p53 was examined in BGC-823 cells, the 5-Aza-CdR-induced inhibition of cell survival was as sensitive as that in A549 cells and triggered apoptosis in a dose-dependent manner. Our data further showed that the cytotoxicity of 5-Aza-CdR was independent of the p53 status in BGC-823 cells. In addition, we observed upregulation of $\mathrm{p} 21^{\mathrm{Waf} 1 / \mathrm{Cip} 1}$ with increased $\mathrm{p} 53$ expression in A549 cells (12), in keeping with a previous observation that $\mathrm{p} 21^{\text {Waf1/Cipl }}$ functions as a downstream target of $\mathrm{p} 53$. Our data suggest that the absence of cell cycle arrest and $\mathrm{p} 21^{\mathrm{Waf} 1 / \mathrm{Cip} 1}$ gene expression differences between 5-Aza-CdR-treated and untreated cells is due to the p53 mutation in BGC-823 cells.

RUNX3 is part of the runt-domain family of transcription factors that act as master regulators of gene expression in major developmental pathways. A more recent study shows that RUNX3 functions as a candidate gastric cancer suppressor gene with hypermethylation at the promoter region (13). Enhancing RUNX3 expression by transfection or other strategies completely abrogated growth advantages and activated apoptosis in gastric cancer (23). In line with previous reports, we observed that $5 \mu \mathrm{M}$ of 5 -Aza-CdR increased RUNX3 expression both at the mRNA and protein levels (24). It is also noteworthy that the expression of RUNX3 was only enhanced at $5 \mu \mathrm{M}$, even though $0.5 \mu \mathrm{M}$ of 5 -Aza-CdR treatment had triggered cell apoptosis. Therefore, 5-Aza-CdR induced apoptosis is, at least in part, due to demethylation of silenced RUNX3. In mammals, global DNA methylation is catalyzed mainly by three DNMTs: DNMT1, DNMT3a and DNMT3b. Recently, increased expression of DNA methyltransferases were shown in various cancer cells (26-28). In vitro studies indicated that $5-\mathrm{Aza}-\mathrm{CdR}$-treated cells were depleted of active DNA methyltransferases through sequestration of the enzyme to azacytosine residues in DNA, resulting in genome-wide demethylation (8). According to our data, although 5-Aza-CdR treatment induced demethylation and expression of RUNX3 the demethylation activity alone could not explain the observed cytotoxicity against BGC-823 cells. We assumed that the cellular effects of 5-Aza-CdR were a direct result of the formation of stable adducts between DNA methyltransferases and 5-Aza-CdR-substituted genomic DNA rather than the DNA hypomethylation. A previous study demonstrated that DNMT1 knockout cells were more resistant to 5-Aza-CdR treatment than that of wild-type cells (29). Also, Oka et al (30) reported that 5-Aza-CdR may mediate DNMT3a and DNMT3b de novo DNA methyltransferases in that DNMT3a and DNMT3b null embryonic stem cells were highly resistant to 5-Aza-CdR when compared to that of wildtype cells. However, our data demonstrate that the levels of DNMT1 and DNMT3a were substantially increased rather than decreased after 5-Aza-CdR treatment $(31,32)$. Our data support the hypothesis, that, cytotoxicity of 5-Aza-CdR results from higher DNA methyltransferase levels. Higher DNA methyltransferase levels were expected to produce more DNA methyltransferase DNA adducts, resulting in an increased response of BGC-823 cells to 5-Aza-CdR. Alternatively, the elevation of DNMT1 and DNMT3a may be not a causative effect but just a side effect of the treatment of the cells with 5-Aza-CdR. We are planning to address this issue in a future study.

In conclusion, our results show that 5 -Aza-CdR, a cytosine analogue designed to inhibit DNMTs, inhibited gastric cancer BGC-823 cell growth via cell apoptosis induced by DNA damage. The upregulation of DNMT1, DNMT3a, and RUNX3 contributed to the cytotoxicity. To our knowledge, this is the first demonstration of p53-independent 5-Aza-CdR action on DNA methyltransferases and demethylation.

\section{Acknowledgements}

This study was supported by a Grant-in-Aid (302140667) from the Wuhan University.

\section{References}

1. Laird PW: Cancer epigenetics. Hum Mol Genet 14: 65-76, 2005.

2. Robertson KD: DNA methylation and human disease. Nat Rev Genet 6: 597-610, 2005.

3. Bender CM, Zingg JM and Jones PA: DNA methylation as a target for drug design. Pharm Res 15: 175-187, 1998.

4. Natsume A, Wakabayashi T, Tsujimura K, Shimato S, Ito M, Kuzushima K, Kondo Y, Sekido Y, Kawatsura H, Narita Y and Yoshida J: The DNA demethylating agent 5-aza-2'-deoxycytidine activates NY-ESO-1 antigenicity in orthotopic human glioma. Int J Cancer 122: 2542-2553, 2008.

5. Glazer RI and Knode MC: 1-beta-D-arabinosyl-5-azacytosine. Cytocidal activity and effects on the synthesis and methylation of DNA in human colon carcinoma cells. Mol Pharmacol 26: 381-387, 1984.

6. Issa JP and Kantarjian H: Azacitidine. Nat Rev Drug Discov Suppl: S6-S7, 2005.

7. Kuendgen A and Lübbert M: Current status of epigenetic treatment in myelodysplastic syndromes. Ann Hematol 87: 601-611, 2008.

8. Haaf T: The effects of 5-azacytidine and 5-azadeoxycytidine on chromosome structure and function: implications for methylationassociated cellular processes. Pharmacol Ther 65: 19-46, 1995.

9. Kiziltepe T, Hideshima T, Catley L, Raje N, Yasui H, Shiraishi N, Okawa Y, Ikeda H, Vallet S, Pozzi S, et al: 5-Azacytidine, a DNA methyltransferase inhibitor, induces ATR-mediated DNA double-strand break responses, apoptosis, and synergistic cytotoxicity with doxorubicin and bortezomib against multiple myeloma cells. Mol Cancer Ther 6: 1718-1727, 2007.

10. Wang C, Zhang Y, Liang J, Shan G, Wang Y and Shi Q: Impacts of ascorbic acid and thiamine supplementation at different concentrations on lead toxicity in testis. Clin Chim Acta 370: 82-88, 2006. 
11. Chai G, Li L, Zhou W, Wu L, Zhao Y, Wang D, Lu S, Yu Y, Wang H, McNutt MA, et al: HDAC inhibitors act with 5-aza-2'deoxycytidine to inhibit cell proliferation by suppressing removal of incorporated abases in lung cancer cells. PLoS One 3: e2445, 2008.

12. Jiemjit A, Fandy TE, Carraway H, Bailey KA, Baylin S, Herman JG and Gore SD: p21 ${ }^{\text {(WAF1/CIP1) }}$ induction by 5 -azacytosine nucleosides requires DNA damage. Oncogene 27: 3615-3623, 2008.

13. Fujii S, Ito K, Ito Y and Ochiai A: Enhancer of zeste homologue 2 (EZH2) down-regulates RUNX3 by increasing histone H3 methylation. J Biol Chem 22: 17324-17332, 2008.

14. Fan H, Zhao ZJ, Cheng YC, Shan YF, Lu ZH, Zhang JQ, Xie W and Fan H: Gene induction and apoptosis in human hepatocellular carcinoma cells SMMC-7721 exposed to 5-aza-2'-deoxycytidine. Chin Med J (Engl) 120: 1626-1631, 2007.

15. Yang H, Hoshino K, Sanchez-Gonzalez B, Kantarjian H and Garcia-Manero G: Antileukemia activity of the combination of 5-aza-2'-deoxycytidine with valproic acid. Leuk Res 29: 739-748, 2005.

16. Gomyo Y, Sasaki J, Branch C, Roth JA and Mukhopadhyay T: 5-aza-2'-deoxycytidine upregulates caspase-9 expression cooperating with p53-induced apoptosis in human lung cancer cells. Oncogene 23: 6779-6787, 2004.

17. Carter BZ, Kornblau SM, Tsao T, Wang RY, Schober WD, Milella M, Sung HG, Reed JC and Andreeff M: Caspaseindependent cell death in AML: caspase inhibition in vitro with pan-caspase inhibitors or in vivo by XIAP or Survivin does not affect cell survival or prognosis. Blood 102: 4179-4186, 2003

18. Shang D, Ito N, Kamoto T and Ogawa O: Demethylating agent 5-Aza-2'-deoxycytidine enhances susceptibility of renal cell carcinoma to paclitaxel. Urology 69: 1007-1012, 2007.

19. Wang H, Zhao Y, Li L, McNutt MA, Wu L, Lu S, Yu Y, Zhou W, Feng J, Chai G, et al: An ATM- and Rad3-related (ATR) signaling pathway and a phosphorylation-acetylation cascade are involved in activation of $\mathrm{p} 53 / \mathrm{p} 21 \mathrm{Waf} 1 / \mathrm{Cip} 1$ in response to 5-aza2'-deoxycytidine treatment. J Biol Chem 283: 2564-2574, 2008.

20. Shi SL, Wang YY, Liang Y and Li QF: Effects of tachyplesin and $\mathrm{n}$-sodium butyrate on proliferation and gene expression of human gastric adenocarcinoma cell line BGC-823. World J Gastroenterol 12: 1694-1698, 2006.

21. Karpf AR, Moore BC, Ririe TO and Jones DA: Activation of the p53 DNA damage response pathway after inhibition of DNA methyltransferase by 5-aza-2'-deoxycytidine. Mol Pharmacol 59: 751-757, 2001.

22. Nieto M, Samper E, Fraga MF, González de Buitrago G, Esteller M and Serrano M: The absence of p53 is critical for the induction of apoptosis by 5-aza-2'-deoxycytidine. Oncogene 23 . $735-743,2004$
23. Nagahama $Y$, Ishimaru M, Osaki M, Inoue T, Maeda A, Nakada C, Moriyama M, Sato K, Oshimura $M$ and Ito $H$ : Apoptotic pathway induced by transduction of RUNX3 in the human gastric carcinoma cell line MKN-1. Cancer Sci 99: 23-30, 2008.

24. Jung Y, Park J, Kim TY, Park JH, Jong HS, Im SA, Robertson KD, Bang YJ and Kim TY: Potential advantages of DNA methyltransferase 1 (DNMT1)-targeted inhibition for cancer therapy. J Mol Med 85: 1137-1148, 2007.

25. Schmelz K, Wagner M, Dörken B and Tamm I: 5-Aza-2'deoxycytidine induces $\mathrm{p} 21^{\mathrm{WAF}}$ expression by demethylation of p73 leading to p53-independent apoptosis in myeloid leukemia. Int J Cancer 114: 683-695, 2005.

26. Zhu YM, Huang Q, Lin J, Hu Y, Chen J and Lai MD: Expression of human DNA methyltransferase 1 in colorectal cancer tissues and their corresponding distant normal tissues. Int J Colorectal Dis 22: 661-666, 2007.

27. Park HJ, Yu E and Shim YH: DNA methyltransferase expression and DNA hypermethylation in human hepatocellular carcinoma. Cancer Lett 233: 271-278, 2006.

28. Roll JD, Rivenbark AG, Jones WD and Coleman WB: DNMT3b overexpression contributes to a hypermethylator phenotype in human breast cancer cell lines. Mol Cancer 7: 15, 2008

29. Jüttermann R, Li E and Jaenisch R: Toxicity of 5-aza-2'-deoxycytidine to mammalian cells is mediated primarily by covalent trapping of DNA methyltransferase rather than DNA demethylation. Proc Natl Acad Sci USA 91: 11797-11801, 1994.

30. Oka M, Meacham AM, Hamazaki T, Rodić N, Chang LJ and Terada N: De novo DNA methyltransferases Dnmt3a and Dnmt3b primarily mediate the cytotoxic effect of 5-aza-2'-deoxycytidine. Oncogene 24: 3091-3099, 2005.

31. Schneider-Stock R, Diab-Assef M, Rohrbeck A, FoltzerJourdainne C, Boltze C, Hartig R, Schönfeld P, Roessner A and Gali-Muhtasib H: 5-Aza-cytidine is a potent inhibitor of DNA methyltransferase $3 \mathrm{a}$ and induces apoptosis in HCT-116 colon cancer cells via Gadd45- and p53-dependent mechanisms. J Pharmacol Exp Ther 312: 525-536, 2005.

32. Palii SS, Van Emburgh BO, Sankpal UT, Brown KD and Robertson KD: DNA methylation inhibitor 5-Aza-2'-deoxycytidine induces reversible genome-wide DNA damage that is distinctly influenced by DNA methyltransferases 1 and 3B. Mol Cell Biol 28: 752-771, 2008. 